\title{
Papel da endotelina no estresse ortostático
}

\section{Endothelin role in the orthostatic stress}

\author{
Luciana Cristina Fosco ${ }^{1}$; Marli Cardoso Martins Pinge ${ }^{2}$
}

\begin{abstract}
Resumo
A adoção da ortostase promove alterações hemodinâmicas. A oposição hidrostática ao retorno venoso, a redução do retorno venoso e a diminuição do débito cardíaco atua como estímulos e geram mecanismos compensatórios. O sistema nervoso central ajusta a atividade autonômica simpática e parassimpática. O sistema nervoso autônomo influencia tônica e reflexamente as principais variáveis do sistema cardiovascular e, juntamente com componentes hormonais, amplia a capacidade de adaptação frente a mudanças posturais. Qualquer dificuldade nesse mecanismo compensatório que impeça seu funcionamento adequado pode resultar em falha da resposta com hipotensão, que, por sua vez, pode levar à hipoperfusão cerebral, hipóxia e perda de consciência. A manutenção da pressão arterial em níveis normais é importante para a homeostasia do meio interno. O barorreflexo desempenha papel fundamental no controle cardiovascular a curto-prazo na adaptação ao estresse ortostático, e prevendo excessivas flutuações da pressão arterial. Durante o estresse ortostático, também ocorrem mudanças neuroendócrinas como alteração nos níveis plasmáticos de endotelina. A endotelina, peptídeo composto de 21 aminoácidos, apresenta potente ação vasoconstritora. Tem sido sugerido um papel para a endotelina, já que está demonstrado que seus níveis no sangue estão aumentados em resposta a um estresse ortostático. Entretanto, embora os níveis de endotelina-1 aumentem em resposta ao estresse postural agudo, seu papel na homeostase cardiovascular e sua relação na liberação de outros hormônios ainda permanecem controversos e bastante desconhecidos em humanos in vivo.
\end{abstract}

Palavras-chave: Endotélio. Hipotensão. Barorreflexo. Intolerância ortostática.

\begin{abstract}
Orthostasis adoption causes hemodynamic changes. Hydrostatic opposition to the venous return, venous return reduction and cardiac output decrease act as stimuli and generate compensatory mechanisms. The central nervous system adjusts the autonomic sympathetic and parasympathetic activity. The autonomous nervous system causes a tonic and reflexive influence on the main variables of the cardiovascular system and, together with hormonal components, extends the adaptation capacity in face of postural changes. Any difficulties in this compensatory mechanism that prevents it from functioning properly may result in hypotension response failure, what, on its turn, can lead to cerebral hypoperfusion, hypoxia and loss of consciousness. Blood pressure maintenance at normal levels is important for the internal medium homeosthasis. Baroreflex plays an important role in the cardiovascular control in the short-term in the adaptation of the orthostatic stress, preventing excessive blood pressure alterations. During the orthostatic stress, neuroendocrinal changes also occur as alteration in endothelin plasma levels. Endothelin, a peptide formed by 21 amino acids, shows a powerful vasoconstrictor action. It also demonstrates that its levels in the blood are increased in response to an orthostatic stress. However, although endothelin-1 levels increase in response to an acute postural stress, its role in cardiovascular homeosthasis and its relation in the release of other hormones are still controversial and quite unknown in humans in vivo.
\end{abstract}

Keywords: Endothelium. Hypotension. Baroreflex. Orthostatic intolerance.

\footnotetext{
1 Especialista em Ciências Fisiológicas.

2 Doutora em Farmacologia. E-mail: martinspinge@uel.br.
} 


\section{Introdução}

A aquisição da posição ortostática, devido à evolução das espécies, trouxe várias implicações para o sistema neurocirculatório (ROCHA, 2006). Acredita-se que o sistema cardiovascular humano se encontre adaptado para manutenção da perfusão cerebral durante a postura ortostática. Embora a força gravitacional crie um gradiente de pressão no sistema circulatório, o homem é capaz de permanecer na postura ereta porque a pressão gravitacional é parcialmente neutralizada por mecanismos que previnem o acúmulo de fluidos nos membros inferiores (ELIAS NETO, 2006).

Mecanismos fisiológicos que atuam de maneira imediata promovem a regulação da pressão arterial no ser humano, que deve adaptar-se constantemente à estimulação diária da gravidade, ou seja, às variações da postura. O sistema nervoso central participa ajustando a atividade autonômica simpática e parassimpática, o que, juntamente com componentes hormonais, possibilita ao animal ou ser humano um melhor desempenho frente a diferentes situações cotidianas (VIEIRA et al., 2006).

A mudança postural é um estímulo fisiológico que ativa o sistema de controle barorreflexo. $\mathrm{O}$ barorreflexo desempenha papel fundamental no controle cardiovascular a curto-prazo; ele promove a adaptação às mudanças ortostáticas e previne excessivas flutuações da pressão arterial (HEUSSER et al., 2005; OLUFSEN; TRAN; OTTESEN, 2006). Os principais sensores envolvidos nesses ajustes são os mecanorreceptores, localizados no seio carotídeo e no arco aórtico (ELIAS NETO, 2006). Esses mecanorreceptores arteriais (ou barorreceptores) são receptores aferentes que respondem a aumentos na pressão vascular transmural. Portanto, as descargas desses neurônios são afetadas por distensibilidade do vaso sangüíneo e alterações no diâmetro absoluto durante mudanças na pressão pulsátil (STEINBACK et al., 2005). Esse sistema, por sua vez, irá ativar os componentes centrais na regulação do sistema cardiovascular e envolve múltiplos componentes do arco barorreflexo (KETCH et al., 2002). Dessa forma, o acúmulo de sangue nos membros inferiores decorrentes do ortostatismo reduz o volume intravascular a nível ventricular e aórtico-carotídeo, o que reduz o tráfico neural aferente oriundo dos barorreceptores provocando aumento do tônus simpático eferente ao coração e à vasculatura periférica. Como resultado, observase o aumento do inotropismo e cronotropismo cardíacos, e a vasoconstrição periférica (COOPER; HAINSWORTH, 2001).

Além da ativação do sistema nervoso simpático, certos agentes humorais são liberados em resposta ao desafio ortostático, e, dentre eles, salientam-se a vasopressina e a angiotensina II, as quais teriam uma participação especial quando as alterações são prolongadas ou quando a pressão arterial começa a cair (MUELLER et al., 2006). Dessa forma, a resposta compensatória ao desafio ortostático utiliza tanto mecanismos neurais quanto humorais para mediar a vasoconstrição e restaurar a pressão arterial.

Anormalidades seletivas do sistema eferente simpático do arco barorreflexo podem ocorrer e geralmente são resultados de alterações nos sistemas compensatórios cardiovasculares, devido às mudanças posturais. Em alguns indivíduos, a falha em secretar noradrenalina em resposta a hipotensão pode resultar em hipotensão ortostática, levando à síncope (desmaio). No entanto, o mais comum é o aparecimento de anormalidades posturais que resultam de condições clínicas denominada intolerância ortostática. Esta é uma síndrome caracterizada por excessiva taquicardia e sintomas adrenérgicos, que ocorrem quando a postura ereta é assumida, como, náusea, dor de cabeça, palpitações, suor excessivo, fadiga, respiração ofegante, tremores entre outros (VAN LIESHOUT; SECHER, 2003; ELIAS NETO, 2006).

A intolerância ortostática representa uma resposta diminuída ao estresse ortostático, os fatores envolvidos na capacidade de compensar a ortostase, 
ou seja, aqueles que possuem ação vasoconstritora, poderiam contribuir para a intolerância ortostática. A literatura tem mostrado que vários fatores podem contribuir para a intolerância ortostática, como, por exemplo, alterações genéticas envolvendo o polimorfismo no gene da NO sintase endotelial (GARLAND; BIAGGIONE; PHILLIPS, 2004), defeito no transportador da noradrenalina (SHANNON; FLATTEM; JORDAN, 2000), alteração na resposta vascular alfa-1 adrenérgica (MECK et al., 2004) e a expressão aumentada do RNA mensageiro para a produção de endotelina (WINKER et al., 2005), entre outros.

A endotelina-1 é um dos mais importantes peptídeos vasoconstritores no sistema vascular humano e participa da manutenção do tônus vasomotor e da pressão arterial (GALLIE; MANES; BRANZI, 2004; MACCARTHY; GROCOTTMASON; PRENDERGAST, 2000), assim como das respostas hemodinâmicas durante o levantar-se (WHITE et al., 1998). Dessa forma, nesta revisão, iremos descrever os efeitos cardiovasculares da endotelina e sua participação no estresse ortostático.

\section{Função da endotelina no sistema cardiovascular}

A endotelina (ET), um peptídeo composto de 21 aminoácidos, é produzida por vários tecidos e apresenta potente ação vasoconstritora (GRANGER, 2003). Foram identificadas três diferentes isoformas de endotelina; endotelina-1 (ET-1), endotelina-2 (ET-2) e endotelina-3 (ET-3), que apresentam diferente distribuição pelos tecidos. A ET-1 está presente no tecido vascular, predominantemente, nas células endoteliais, células renais, endometriais, pulmonares, mamárias entre outras, e também é secretada por neurônios e por astrócitos do sistema nervoso central. A ET-2 exibe uma distribuição menos ampla, sendo encontrada somente no tecido renal e no intestino. A ET-3 está presente no cérebro, rins, intestino, pulmões e glândula supra-renal (PALMA et al., 2002).
As endotelinas exercem seus efeitos por meio da ativação de receptores acoplados à proteína $\mathrm{G}$. Esses receptores são encontrados em muitos tecidos e órgãos, incluindo a parede dos vasos sanguíneos, músculo cardíaco, sistema nervoso central, pulmões e rins (KATZUNG, 2003).

Foram identificados três subtipos de receptores: ETA, ETB, e ETC. O subtipo ETA apresenta uma grande afinidade por ET-1, enquanto o ETC exibe alta afinidade por ET-3. O receptor ETB demonstra afinidades similares pelos três peptídeos. (POULAT; COUTURE, 1998). Nas paredes dos vasos sanguíneos, os receptores ETA são expressos principalmente por células musculares lisas enquanto que, o subtipo ETB é expresso por células endoteliais e células musculares lisas. O receptor ETC ainda não foi demonstrado em tecidos de mamíferos (KATZUNG, 2003). Quando os receptores ETA são ativados, promovem vasoconstrição. Já os receptores ETB são subdivididos em: ETB1, que está presente no endotélio, e promove vasodilatação pela estimulação da produção de óxido nítrico e, ETB2, que se manifesta nas células musculares lisas e provocam vasoconstrição. (RANG; DALE; PITTER, 2001)

A produção de endotelina é regulada a nível transcricional e, quando liberada, atua de maneira autócrina e parácrina em células próximas, como as células musculares lisas (YANAGISAWA et al., 1988; RUBANYL; POLOKOFF, 1994; PARRIS; WEEB, 1997). Vários fatores agem estimulando a produção e liberação de ET, incluindo plaquetas ativadas, endotoxinas, trombina, citocinas, angiotensina, adrenalina, insulina, hipóxia e arginina-vasopressina. Os fatores que agem inibindo a liberação de ET incluem óxido nítrico, prostaglandinas E2 e I2, heparina e peptídeo natriurético (RANG; DALLE; PITTER, 2001).

As endotelinas causam vasoconstrição dosedependente nas células do tecido vascular. Quando a endotelina é administrada por via intravenosa, causa uma rápida e transitória redução da pressão arterial, 
seguida de um aumento prolongado. A resposta depressora resulta da liberação de prostaciclina e de óxido nítrico do endotélio vascular, enquanto a resposta pressora decorre da constrição direta do músculo liso vascular. As endotelinas também agem sobre o coração promovendo vasoconstrição coronariana, atuam sobre os pulmões e causam potente constrição do músculo liso traqueal e brônquico. Elas também agem sobre os rins, causando vasoconstrição e diminuição da taxa de filtração glomerular e da excreção de sódio e de água (KATZUNG, 2003). Estes efeitos renais da endotelina sobre a excreção diminuída de sódio parecem envolver uma ação no canal de sódio sensível à amilorida e/ou atividade da Na/K ATPase no duto coletor (GE et al., 2006).

A endotelina também promove efeitos pressores atuando no sistema nervoso central, já que, após sua administração no ventrículo lateral, ocorre aumento da pressão arterial, o qual é mediado por um aumento do fluxo simpático (GULATI; REBELLO; KUMAR, 1997). Sua ação sistêmica e intracerebroventricular pode ocorrer por meio de interações com os órgãos circunventriculares, como o órgão subfornical, o qual possui abundantes quantidades de receptores de endotelina (ROSEKI et al., 1989). Além disso, vários estudos têm demonstrado que a endotelina 1 estimula a secreção de vasopressina, tanto in vivo quanto in vitro (ROSSI, 1993).

Níveis plasmáticos elevados de endotelina foram encontrados em grupos de pacientes com hipertensão essencial e hipertensão induzida por ciclosporina. No entanto, a elevação do nível plasmático de endotelina não leva , necessariamente, a aumento sustentado da pressão arterial. Infusão crônica de endotelina em ratos não produz hipertensão sustentada, o mesmo ocorrendo com ratos transgênicos, produzidos pela adição de cópia do gen ET-2 humano da endotelina. Isso indica que a elevação dos níveis plasmáticos de endotelinas na hipertensão arterial essencial e na hipertensão induzida por ciclosporina exerce apenas papel coadjuvante na patogênese ou se elevam, secundariamente, durante o estabelecimento do processo hipertensivo (KRIEGER; FRANCHINI; KRIEGER, 1996).

\section{Participação da endotelina no estresse ortostático}

As adaptações cardiovasculares em resposta ao estresse ortostático, como os ajustes dos determinantes da pressão arterial dependem, portanto, de mecanismos complexos que envolvam o sistema nervoso autônomo e sistemas humorais.

Estudos sugerem que a endotelina-1 pode ter papel compensatório na manutenção da pressão arterial durante as mudanças posturais, e pode também estar envolvida no mecanismo da síncope neuralmente mediada (MEHTA et al, 1995). Os níveis de endotelina-1 aumentam em resposta ao estresse postural (STEWART; CERNACEK; COSTELLO, 1992), e em outros estudos foi sugerido que a liberação diminuída de endotelina levaria à hipotensão ortostática (KAUFMANN; ORIBE; OLIVER, 1991) e à síncope neurocardiogênica (WHITE et al., 1998).

Mehta et al. (1995) utilizaram o teste de inclinação (head-up tilt) para avaliar o papel da ET-1 em crianças com síncope. No estudo, todas as crianças com síncope apresentaram elevados níveis basais de endotelina, quando comparadas às crianças saudáveis (grupo controle). Crianças com síncope e resposta negativa ao teste de inclinação apresentaram os níveis basais de ET-1 mais altos, quando comparadas a crianças com resposta positiva ao teste e ao grupo controle. Essas crianças não mostraram aumentos nos níveis de endotelina em resposta ao estresse ortostático. Isso pode sugerir que haja uma alteração no tônus basal que as proteja do estresse ortostático, provocado pelo teste de inclinação, embora o balanço neuro-humoral permaneça anormal.

Embora a ET-1 seja um sinalizador da resposta ao teste de inclinação em crianças com síncope servindo como um marcador para identificar indivíduos com 
síncope, segundo Mehta et al. (1995), não está claro se ela constitui a causa principal ou apenas um reflexo de outra anormalidade.

Magerkurth, Riedel e Braune (2005) encontraram níveis séricos de endotelina significativamente elevados durante o repouso e durante o teste de inclinação em indíviduos com síncope, quando comparados a indivíduos saudáveis. Os níveis de endotelina foram maiores no grupo com síncope, independente desses indivíduos terem apresentado síncope ou não por ocasião do teste. Esses resultados apontam para uma liberação permanentemente aumentada de endotelina nos indivíduos com tendência para a sincope vasovagal, independente do estresse ortostático, pois os níveis de endotelina mostraram-se similares na posição supina e em pé.

Também em pacientes com insuficiência cardíaca congestiva, os níveis basais de endotelina estão elevados ao repouso e não aumentam ao teste de inclinação (MEHTA et al., 1995), o que contrasta com indivíduos saudáveis, para os quais há um aumento nos níveis de endotelina com o "headup tilt” (STEWART; CERNACEK; COSTELLO, 1992).

White et al. (1998) avaliaram as mudanças na liberação de neuro-hormônios vasoconstritores e ET-1, em indivíduos tolerantes comparados com indivíduos com síncope induzida por inclinação ao repouso e durante o teste de inclinação. Os níveis de ET-1 aumentaram consistentemente em resposta ao head-up tilt em indivíduos capazes de tolerar o teste. Em contraste, indivíduos com síncope não exibiram aumento significativo nos níveis de ET-1, durante o tilt ou imediatamente antes da síncope. De acordo com White et al. (1998), a liberação bifásica da ET-1 durante o teste sugere que a ET-1 pode desempenhar um papel na manutenção do tônus vasomotor durante o estresse ortostático agudo. Essas observações estão de acordo com Stewart, Cernacek e Costello (1992), que relatatram a mesma resposta bifásica da ET-1 em indivíduos normais. A ausência de aumento significativo na ET-1 durante o teste pode ter influência na inabilidade para suportar o estresse ortostático. (WHITE et al., 1998)

O ortostatismo de longo prazo tem grande impacto no sistema vascular, especialmente nas extremidades inferiores. Raffai et al. (2005) investigaram vesículas eletro-densas no endotélio vascular quanto a sua constituição, distribuição arteriovenosa e a resposta ao estresse ortostático crônico de vasos de extremidade. A endotelina e fator de crescimento derivado de plaqueta foram identificados nas vesículas. Raffai et al. (2005) concluíram que as vesículas densas no endotélio de vasos de extremidade podem representar um sistema secretório vesicular, ou de armazenamento de endotelina e fator de crescimento derivado de plaquetas que participam na adaptação vascular regional à carga ortostática em longo prazo.

\section{Conclusões}

A tolerância ortostática parece ser determinada, pelo menos em parte, pela capacidade do sistema cardiovascular em promover vasoconstrição. Durante as alterações posturais, tem sido sugerido um papel para a endotelina, baseado nos achados de que a liberação diminuída desta ocorre em indivíduos com hipotensão ortostática e síncope neurocardiogênica. Dessa forma, é possível que a endotelina deve contribuir para a vasoconstrição necessária para manter a pressão arterial durante a postura ereta.

Entretanto, seu papel na homeostase cardiovascular e sua relação na liberação de outros hormônios ainda permanecem bastante desconhecidos em humanos in vivo.

\section{Referências}

COOPER, V.L.; HAINSWORTH, R. Carotid baroreceptor reflexes in humans during orthostatic stress. Experimental Physiology, New York, v. 86, n. 5, p. 677-681, 2001. 
ELIAS NETO, J. Great arteries contribution in orthostasis cardiovascular adaptation. Arquivos Brasileiros de Cardiologia, São Paulo, v. 87, n. 2, ago. 2006.

GALLIE, N.; MANES, A.; BRANZI A. The endothelin systeminpulmonaryarterialhypertension. Cardiovascular Research, London, v. 61, n. 2, p. 227-237, 2004.

GARLAND, E. M.; BIAGGIONE, I.; PHILLIPS, J. The T-786c and Glu298asp polymorfisms of the endothelial nitric oxide synthase gene are associated with postural tachycardia syndrome. Hypertension, Dallas, v. 44, n. 1, p. 100-107; 2004.

GE, Y.; BAGNALL, A.; STRICKLETT, P. K.; STRAIT, K.; WEBB, D. J.; KOTELEVTSEV, Y.; KOHAN, D. E. Collecting duct-specific knockout of the endothelin $\mathrm{B}$ receptor causes hypertension and sodium retention. American Journal of Physiology, Baltimore, v. 291, n. 6, p. F1274-F1280, 2006.

GRANGER, J. P. Endothelin. American Journal of Physiology: Regulatory, Integrative and Comparative Physiology, Bethesda, v. 285, n. 2, p. R298-R301, 2003.

GULATI, A.; REBELlO, A.; KUMAR, A. Role of sympathetic nervous system in cardiovascular effects of centrally administered endothelin-1 in rats. American Journal of Physiology, Baltimore, v. 273: H1177-H1186, 1997.

HEUSSER, K.; TANK, J.; LUFT, F. C.; JORDAN, J. Baroreflex Failure. Hypertension, Dallas, v. 45, n. 5, p. 834-839, 2005.

KATZUNG, B. G. Farmacologia básica e clínica. Rio de Janeiro: Guanabara Koogan, 2003. p. 266-268.

KAUFMANN, H; ORIBE, E; OLIVER, J. A. Plasma endothelin during upright tilt: relevance for orthostatic hypotension? Lancet, London, v. 338, n. 8782-3, p. 1542$1545,1991$.

KETCH, T.; BIAGGIONI, I.; ROBERTSON, R.; ROBERTSON, D. Four Faces of Baroreflex Failure. Circulation, Baltimore, v. 105, n. 21, p. 2518-2523, 2002.

KRIEGER, E. M.; FRANCHINI, K. G.; KRIEGER, J. E. Pathophysiology of primary hypertension. Medicina, Ribeirão Preto, v. 29, n. 2/3, p. 181-192, Apr./Sep. 1996.

MACCARTHY, P. A.; GROCOTT-MASON, R.; PRENDERGAST, B. D. Contrasting inotropic effects of endogenous endothelin in the normal and failing human heart: studies with an intracoronary ET(a) receptor antagonist. Circulation, Baltimore, v. 101, n. 2, p. 142$147,2000$.

MAGERKURTH, C.; RIEDEL, A.; BRAUNE, S. O. Permanent increase in endothelin serum levels in vasovagal syncope. Clinical Autonomic Research, Oxford, v. 15, n. 4, p. 299-301, Aug. 2005.

MECK, J. V.; WATERS, W. W.; ZIEGLER, M. G.; BLOCK, H. F.; MILLS, P. J.; ROBERSTSON, D.; HUANG, P. L. Mechanisms of postspaceflight orthostatic hypotension: low alpha-1 adrenergic receptor before flight and central autonomic dysregulation postflight. American Journal of Physiology, Baltimore, v. 286, n. 4, p. H486-H495, 2004.

MEHTA, M.; WOLFF, G.; YOUNG, M. L.; MAS, M. S.; ESCOBAR, A.; GELBAND, H. Usefulness of endothelin-1 as a predictor of response to head-up tilttable testing in children with syncope. The American Journal of Cardiology, New York, v. 76, n. 1, p. 86-88, 1995.

MUELLER, P. J.; SULLIVAN, M. J.; GRINDSTAFF, R. R.; CUNNINGHAM, J. T.; HASSER, E. M. Regulation of plasma vasopressin and renin activity in conscious hindlimb-unloaded rats. American Journal of Physiology, Baltimore, v. 291, n. 1, p. R46-R52, 2006.

OLUFSEN, M. S., TRAN, H. T.; OTTESEN, J. T. Modeling baroreflex regulation of heart rate during orthostatic stress. American Journal of Physiology, Baltimore, v. 291, n. 5, p. 1355-1368, 2006.

PALMA, B. D.; GABRIEL JÚNIOR, A.; BIGNOTTO, M.; TUFIK, S. Paradoxical sleep deprivation increases plasma endothelin levels. Brazilian Journal of Medical and Biological Research, Ribeirão Preto, v. 35, n. 1, p. 75-79, 2002.

PARRIS, R. J.; WEBB, D. J. The endothelin system in cardiovascular physiology and pathophysiology. Vascular Medicine, London, v. 2, n. 1, p. 31-43, 1997.

POULAT, P.; COUTURE, R. Increased pulmonary vascular permeability and edema induced by intrathecally injected endothelins in rat. European Journal of Pharmacology, Amsterdam, v. 344, n. 2-3, p. 251-259, 1998.

RAFFAI, G.; FEHÉRB, E.; NÁDASYA, G.; PAKUC, S.; POGÁNYC, G.; TÍMÁRC, F.; SZENDEC, B.; MONOSA, E. Selective Suppression of an Endothelin and Platelet-Derived Growth Factor Containing Vesicular System in Endothelium of Rat Saphenous Vein by LongTerm Orthostasis. Journal of Vascular Research, Basel, v. 42, n. 2, p. 157-164, 2005.

RANG,H.P.; DALE, M. M.;PITTER, J.M.Farmacologia. Rio de Janeiro: Guanabara Koogan, 2001. p. 232-233.

ROCHA, E. A. Síndromes neuralmente mediadas. Arquivos Brasileiros de Cardiologia, São Paulo, v. 87, n. 3, p. e34-e44, Sep. 2006. 
ROSEKI, C.; IMAI, M.; HIRATA, Y.; YANAGISAWA, M.; MUSAKI, T. Autoradiographic distribution in rat tisúes of binding sites for endothelin : a neuropeptide? American Journal of Physiology, Baltimore, v. 256, n. 4, p. R858-R866, 1989.

ROSSI, N. F. Effect of endothelin 3 on vasopressin release in vitro and water excretion in vivo in Long Evans rats. Journal of Physiology, Paris, v. 461, n. 1, p. 501-511, 1993.

RUBANYI, G. M.; POLOKOFF, M. A Endothelins: molecular biology, biochemistry, pharmacology, physiology, and pathophysiology. Pharmacological Reviews, Baltimore, v. 46, n. 3, p. 325-415, 1994.

SHANNON, J. R.; FLATTEM, N. L.; JORDAN, J. Orthostatic intolerance and Tachycardia associated with Norepinephryne-transporter deficiency. New England Journal of Medicine, Waltham, v. 342, n. 8, p. 541-549, 2000.

STEINBACK, C. D.; O’LEARY, D. D.; BAKKER, J.; CECHETTO, A. D.; LADAK, H. M.; SHOEMAKER J. K. Carotid distensibility, baroreflex sensitivity, and orthostatic stress. Journal of Applied Physiology, Bethesda, v. 99, n. 1, p. 64-70, 2005.

STEWART, D. J.; CERNACEK, P.; COSTELLO, K. B. Elevated endothelin-1 in heart failure and loss of normal response to postural change. Circulation, Baltimore, v. 85 , n. 2, p. 510-517, 1992.
VAN LIESHOUT, J. J.; SECHER, N. H. Reflex control of sympathetic vasoconstrictor activity in vasovagal syncope. Clinical Autonomic Research, Oxford, v. 13, n. 3, p. 175-177, 2003.

VIEIRA, A. A; DE PAULA, P. M.; DE LUCA JÚNIOR, L. A.; COLOMBARI, D. A. S.; COLOMBARI, E.; MENANI, J. V. Importância da região anteroventral do terceiro ventrículo (AV3V) no controle cardiovascular e do equilíbrio hidroeletrolítico. Medicina, Ribeirão Preto, v. 39, n. 1, p. 21-27, 2006.

WHITE, M; CERNACEK P; COURTEMANCHE M; STEWART D; TALAJIC M; MIKES E; VANTRIMPONT P; BUSSIÈRES L; ROULEAU, J. L. Impaired Endothelin-1 release in tilt-induced syncope. The American Journal of Cardiology, New York, v. 81, n. 5, p. 460-464, 1998.

WINKER, R.; GARLAND, E. M.; RUDIGER, H. W.; DIEDRICH, A.; BIAGGIONI, I.; PONOCNY, I.; CASCORBI, I.; ROBERTSON, D. Influence of an insertion variant in the 5'UTR of the endothelin-1 gene on orthostatic intolerance. The American Journal of Medical Science, Charleston, v. 330, n. 4, p. 166-171, Oct. 2005.

YANAGISAWA, M.; KURIHARA, H.; KIMURA, S.; TOMOBE, Y.; KOBAYASHI, M.; MITSUI, Y.; YAZAKI, Y.; GOTO, K.; MASAKI, T. A novel potent vasoconstrictor peptide produced by vascular endothelial cells. Nature, London, v 332, n. 6163, p. 411-415, 1988. 
